\title{
Somatic, Endurance Performance and Heart Rate Variability Profiles of Professional Soccer Players Grouped According to Age
}

\author{
by \\ Michal Botek ${ }^{1}$ Jakub Krejčí1, Andrew J. McKune ${ }^{2,3}$, Iva Klimešová ${ }^{1}$
}

\begin{abstract}
This cross-sectional study compared somatic, endurance performance determinants and heart rate variability (HRV) profiles of professional soccer players divided into different age groups: GI (17-19.9 years; $n=23)$, GII (20-24.9 years; $n=45)$, GIII (25-29.9 years; $n=30)$, and GIV (30-39 years; $n=26)$. Players underwent somatic and HRV assessment and maximal exercise testing. $H R V$ was analyzed by spectral analysis of $H R V$, and high (HF) and low (LF) frequency power was transformed by a natural logarithm (Ln). Players in GIV (83 $\pm 7 \mathrm{~kg})$ were heavier $(p<0.05)$ compared to both GI $(73 \pm 6 \mathrm{~kg})$, and GII $(78 \pm 6 \mathrm{~kg})$. Significantly lower maximal oxygen uptake $(V O 2 \mathrm{max}, \mathrm{ml} \bullet \mathrm{kg}$ -

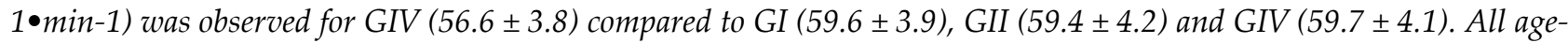
groups, except for GII, demonstrated comparable relative maximal power output (Pmax). For supine HRV, significantly lower Ln HF (ms2) was identified in both GIII (7.1 \pm 0.8$)$ and GIV (6.9 \pm 1.0$)$ compared to GI (7.9 \pm 0.6$)$ and GII (7.7 \pm 0.9). In conclusion, soccer players aged $>25$ years showed negligible differences in Pmax unlike the age group differences demonstrated in VO2max. A shift towards relative sympathetic dominance, particularly due to reduced vagal activity, was apparent after approximately 8 years of competing at the professional level.
\end{abstract}

Key words: body composition, maximal oxygen uptake, vagal activity, sports career length, aging.

\section{Introduction}

Soccer is an intermittent and high intensity team sport (Rampinini et al., 2007), where strength and endurance are considered important co-factors for game performance (Hoff and Helgerud, 2004). Previously, Pate and Kiska (1984) introduced the concept of three major factors responsible for inter-individual variability in aerobic endurance performance, namely maximal oxygen uptake (VO2max), the anaerobic threshold and work economy. High $\mathrm{VO} 2 \mathrm{max}$ is acknowledged to play an important role in recovery (lactate turn over) between the exhaustive power-demanding sprints and high intensity periods during soccer match play (Helgerud et al., 2011). However, VO2max is less sensitive to adaptations to training compared with the anaerobic threshold (Hoff and Helgerud, 2004). Among individuals with similar VO2max values, endurance performance may vary significantly with the difference possibly related to better anaerobic capacity or work economy (Helgerud, 1994). Interestingly, strength and plyometric training have been shown to improve work economy and in turn endurance performance (Hoff and Helgerud, 2004).

It is common that professional soccer

\footnotetext{
1 - Department of Natural Sciences in Kinanthropology, Faculty of Physical Culture, Palacký University Olomouc, Olomouc, Czech Republic.

2- Discipline of Sport and Exercise Science, Faculty of Health, UC-Research Institute for Sport and Exercise, University of Canberra, ACT, Australia.

3- Discipline of Biokinetics, Exercise and Leisure Sciences, School of Health Sciences, University of KwaZulu-Natal, Durban, South Africa.
} 
teams consist of players of a wide age range. However, it is well documented that biological function and physical performance reach their peak at 30 years of age (Brooks et al., 2004). Thereafter, there is a natural decrement of $\sim 10 \%$ per decade for $\mathrm{VO} 2 \mathrm{max}$ due to reductions in both a maximal heart rate (HRmax) and muscle mass (Hawkins and Wiswell, 2003). On the other hand, some studies have shown that the deterioration in VO2max with aging in well trained endurance athletes up to 50 years old is either minimal (Pimentel et al., 2003) or equal to $\sim 0.5 \%$ VO2max per year in athletes between the 23-45 years of age (Trappe et al., 1996). For example, elite soccer players aged 17-36 years had similar VO2max and ventilatory anaerobic threshold (VT) values (Signorelli et al., 2012).

A somatic profile also plays an important part in soccer performance (Gil et al., 2007), with somatic variables, particularly body height $(\mathrm{BH})$, varying between playing positions considerably (Reilly and Williams, 2003). Body fat percentage (\%BF) typically varies among national senior soccer teams (7 to $12 \%$ ) (Reilly and Williams, 2003), whereas in junior soccer players (17-19 years old) fat content values are more homogeneous (7\%) (Arroyo et al., 2008). Trappe et al. (1996) reported an elevated fat content in senior endurance trained athletes with no changes in fat free mass (FFM). In addition, Tessitore et al. (2005) reported a significantly elevated \%BF with a mean value of 25 in senior soccer players aged between 53 and 72 years.

Currently, the functional changes in autonomic nervous system (ANS) activity in response to training are considered a promising training status indicator (Botek et al., 2014b; Buchheit, 2014). From a medical standpoint, a reduction in a cardiac vagal outflow and/or a relative increase in sympathetic activity are associated with an increased risk of cardiovascular disease development, including hypertension, cardiac arrhythmias, and/or sudden cardiac death (Billman 2009; Vanoli et al., 1991). It has been shown repeatedly that regular aerobic training induces an adaptation-related increase in resting vagal activity (De Meersman and Stein, 2007; Sandercock et al., 2005), and physical activity may also have a decelerating effect relating to the ageing of cardiac related vagal functioning (Aubert at al., 2003; Banach et al.,
2000). Nevertheless, professional soccer players over their sports career are frequently faced with high physical and mental stressors that may represent a chronic allostatic load that has been associated with decreased vagal activity (Thayer and Sternberg, 2006). In this regard, one would expect some negative outcomes related to autonomic cardiac function in older players. Spectral analysis (SA) of heart rate variability (HRV), the beat-to-beat fluctuation of the heart rate (HR), is commonly accepted as a noninvasive method to determine ANS activity (Akselrod et al., 1981). The ANS activity is typically monitored in a supine position, standing, and/or during an orthoclinostatic stress test, with the orthostatic challenge reducing the vagal outflow and stimulating a sympathoadrenal response (Eckberg, 1997), whereas clinostasis induces a rise in vagal activity and a decline in the HR (Task Force, 1996).

Therefore, the main purpose of this study was to compare selected somatic, endurance performance determinants (VO2max, VT and maximal power output (Pmax)), and HRV profiles of professional soccer players divided into different age groups (age range 17-39 years old).

\section{Material and Methods}

\section{Participants}

This study included 153 soccer players who were playing either in the first junior or senior Czech Republic soccer league, and had undergone pre-season laboratory testing during the period between 2010 and 2014 in our facility. None of the soccer players had previously participated in the European championships. Inclusion criteria for participation in this study were the following: each player was to i) participate in the entire laboratory testing protocol; ii) be free of any health problems that may have limited performance in any laboratory test; iii) be a member of the senior team squad at the time of testing. Based on these criteria, 8 players from the U19 category who were playing in the junior league, but practicing with the senior squad were included; furthermore, 29 players were excluded as they did not meet the expected requirements. This research was performed in accordance with the ethical guidelines outlined in the Declaration of Helsinki and was approved by the Ethics Committee of the Faculty of Physical Culture, 
Palacký University Olomouc. All of the players participating in the study were volunteers and had given their written informed consent before the commencement of the study.

\section{Testing procedures}

The entire testing protocol was performed between 8-12 am on a single day in the following order: a resting 10-lead electrocardiogram (ECG) assessment (Delta 60D, Cardioline, Cavareno, Italy); a resting autonomic cardiac activity assessment; basic anthropometrical measurements; and an incremental running test. Considering the purpose of the current study, only age and data related to anthropometry, autonomic cardiac activity and aerobic endurance capacity were included.

\section{Autonomic cardiac activity assessment}

Monitoring of autonomic cardiac activity was performed between 8:00 and 10:00 am in a laboratory where the ambient temperature ranged from 22 to $24{ }^{\circ} \mathrm{C}$. Testing of HRV during the morning followed the recommendation of Malik and Camm (1995) who highlighted the importance of considering circadian oscillations in HRV (Mølgaard et al., 1991). During the measurement, each player was shielded from acoustic and visual disturbances. To determine resting HR and HRV variables, the ECG signal was measured at a sampling frequency of $1000 \mathrm{~Hz}$ using VarCor PF7 (DIMEA Group, Olomouc, Czech Republic). ECG sampling was performed during an orthoclinostatics maneuver (supinestanding-supine) up to January 2013, and in the following period, a time-modified orthoclinostatics test was applied, as both orthoclinostatics challenges provide comparable HRV results (Botek et al., 2013). The ECG record was examined and all premature ventricular contractions, missing beats and any artefacts were manually filtered. A set of 300 artefact-free subsequent RR intervals was obtained from each phase. SA HRV was used to assess the autonomic cardiac activity and the power spectral density curve of the collected signals was estimated using the Fast Fourier Transform method with a partly modified Coarse-Graining Spectral Analyses algorithm (Yamamoto and Hughson, 1991). Two spectral frequency bands were analyzed: high frequency $(\mathrm{HF})$ power $(0.15$ to $0.50 \mathrm{~Hz})$ that is thought to represent respiratory related cardiac vagal activity and low frequency (LF) power (0.05 to $0.15 \mathrm{~Hz}$ ) that is considered to exhibit baroreflex activity together with a sympathetic and vagal outflow (Task Force, 1996), whereas the LF/HF ratio reflects the sympathovagal balance (Malliani et al., 1991). A time domain variable, the root mean square of successive difference of $R R$ intervals (RMSSD) was also used. RMSSD is regarded as an index of vagal activity (Buchheit, 2014) that is thought to be resistant to the effects of breathing frequency (Penttilä et al., 2001).

\section{Anthropometrical measurements}

The soccer players had their $\mathrm{BH}$ and body weight (BW) measured using the SOEHNLE 7307 (Leifheit, Nassau, Germany). The \%BF was determined using bioimpedance analysis (Tanita BC-418 MA, Tanita, Tokyo, Japan).

\section{Incremental running test}

Each player underwent an incremental running test on a treadmill (Lode Valiant, Groningen, Netherlands) in order to determine $\mathrm{VO}_{2 \max }$ and $\mathrm{HR}_{\max }$. The exercise protocol consisted of a $4 \mathrm{~min}$ warm-up ( $2 \mathrm{~min}$ at $8 \mathrm{~km} \cdot \mathrm{h}^{-1}$ with $0 \%$ inclination and a further $2 \mathrm{~min}$ at the same speed with a 5\% inclination). The speed was then increased to $10 \mathrm{~km} \cdot \mathrm{h}^{-1}$ for $1 \mathrm{~min}$ with the gradient kept at 5\%. For each minute thereafter speed increased by $1 \mathrm{~km} \cdot \mathrm{h}^{-1}$ with the gradient at $5 \%$ up to maximal speed of $16 \mathrm{~km} \cdot \mathrm{h}-1$. From this stage only inclination increased by $2.5 \%$ every min until exhaustion. Breath-by-breath ventilation and gas exchange were continuously analyzed (Ergostik, Geratherm Respiratory, Bad Kissingen, Germany) during the exercise with the data averaged to $30 \mathrm{~s}$ for further analysis. Gas and flow analyzers were recalibrated before and after each testing using gases of known concentration and a 31 calibration syringe. During the test, the ambient temperature was maintained between 20 and $24{ }^{\circ} \mathrm{C}$, and relative humidity between 40 and $60 \%$. The following criteria were used to document that $\mathrm{VO}_{2 \max }$ was achieved: i) lack of an increase in $\mathrm{VO}_{2}$ upon an increase in the work rate, ii) a respiratory exchange ratio $>1.10$ (Shephard and Åstrand, 1992). $\mathrm{VO}_{2 \max }$ was considered the highest $\mathrm{VO}_{2}$ value in the final 30 seconds of the test (Millet et al., 2003). The VT level was assessed based on selected ventilatory variable $\left(\mathrm{VE}, \mathrm{VE} / \mathrm{VO}_{2}\right.$ and $\mathrm{VE} / \mathrm{VCO}_{2}$ ratios) responses according to Wasserman et al. (1987). HR responses (Polar, Kempele, Finland) were monitored continuously during the incremental running test. The accuracy 
of the Ergostik measurement device expressed as a $95 \%$ confidence interval is $\pm 3 \%$ (datasheet of Geratherm Company). Consequently, the technical error expressed as standard deviation was $3 \% / 1.96=1.5 \%$. Therefore, the minimal detectable change (MDC) for $\mathrm{VO}_{2}$ measurement was calculated as MDC $=1.5 \% * 1.96 * \sqrt{2}=4.2 \%$ (Hopkins, 2000).

$P_{\max }$ was established indirectly according to the following formula (American College of Sports Medicine, 1986):

$P=(0.2 s+0.9 s g+3.5) / 10.5$

where: $P$ is relative power output $\left(\mathrm{W} \cdot \mathrm{kg}^{-1}\right), s$ is speed $\left(\mathrm{m} \cdot \mathrm{min}^{-1}\right)$ and $g$ is fractional grade (unitless).

\section{Statistical analysis}

The players were divided into four age groups: GI (17-19.9 years), GII (20-24.9 years), GIII (25-29.9 years), and Giv (30-39 years). According to Signorelli et al. (2012), the chi-square test was used to determine the relationship between age and a player position and no significant relationship was revealed. A one-way analysis of variance (ANOVA) was used to evaluate the differences between the age groups. Pairwise multiple comparisons were performed using the Tukey's post-hoc test. The Pearson's coefficient of correlation (r) was calculated to assess the linear dependence between two selected variables. Normal distribution of the analyzed data was checked using the Kolmogorov-Smirnov test. BH, body mass index (BMI), \%BF, FFM, VT, $\mathrm{VO}_{2 \max }$, absolute maximal oxygen uptake ( $\mathrm{VO}_{2 m a x a b s,}$ expressed as $\left.1 \cdot \mathrm{min}^{-1}\right)$ and resting heart rate ( $\left.\mathrm{HR}_{\text {rest }}\right)$ data were normally distributed. BW $(p=0.007)$, $P_{\max }(p=0.001)$ and $\operatorname{HR}_{\max }(p=0.004)$ were not normally distributed. After visual inspection of the data distribution, both variables were processed untransformed as ANOVA is considered a robust test against such violations of normality. Since HRV variables differed considerably from normal distribution, a natural logarithm (Ln) transformation was applied. The statistical significance was set at $p<0.05$. The practical significance of differences in means between groups was assessed using effect sizes (ES) calculated as Cohen's d. The criteria used to interpret the ES were: $\leq 0.2$ trivial, $>0.2-0.6$ small, $>0.6-1.2$ moderate, $>1.2$ large (Hopkins et al., 2009). Data were expressed as means \pm standard deviation and mean difference $(\Delta)$ with a $95 \%$ confidence interval. Statistical analyses were performed using the STATISTICA 12.0 (StatSoft, Tulsa, OK, USA).

\section{Results}

Based on the ANOVA results (Table 1), the players were significantly heavier in GII $(\Delta=5 \mathrm{~kg}$, $95 \%$ confidence interval $[1 ; 10]$, ES $=0.57)$, GIII $(\Delta=$ $6[2 ; 11], \mathrm{ES}=0.69)$, and Giv $(\Delta=10[5 ; 15], \mathrm{ES}=$ 1.10) compared to Gi and the players in Giv $(\Delta=5$ $[1 ; 9], \mathrm{ES}=0.52)$ were significantly heavier than GII. BMI $\left(\mathrm{kg} \cdot \mathrm{m}^{-2}\right)$ was significantly higher in GII $(\Delta$ $=1.3[0.4 ; 2.2], \mathrm{ES}=0.68)$, GIII $(\Delta=1.8[0.8 ; 2.8]$, ES $=0.95)$, and GIV $(\Delta=2.0[1.0 ; 3.0], \mathrm{ES}=1.05)$ compared to Gi. \%BF was significantly higher in GIII $(\Delta=2.7[0.9 ; 4.5], \mathrm{ES}=0.78)$ and GIV $(\Delta=3.8$ $[2.0 ; 5.6], \mathrm{ES}=1.10)$ compared to Gi and also significantly higher in GIv $(\Delta=2.3[0.7 ; 3.8]$, ES = $0.66)$ compared to GII. FFM (kg) was significantly higher in GII $(\Delta=4[0 ; 7], \mathrm{ES}=0.48)$ and GIV $(\Delta=6$ $[2 ; 10], \mathrm{ES}=0.81)$ compared to GI.

Table 1 shows that there was significantly lower $\mathrm{VO}_{2 \max }\left(\mathrm{ml} \cdot \mathrm{kg}^{-1} \cdot \mathrm{min}^{-1}\right)$ for Giv compared to GI $(\Delta=-3.1[-6.1 ;-0.1], \mathrm{ES}=0.53), \mathrm{GII}(\Delta=-2.8[-5.4$; $-0.2], \mathrm{ES}=0.49)$ and $\mathrm{G}$ III $(\Delta=-3.1[-5.9 ;-0.2], \mathrm{ES}=$ $0.54)$. $\mathrm{VO}_{2 \text { maxabs }}\left(1 \cdot \mathrm{min}^{-1}\right)$, was significantly lower for Gi compared to GIII and GIv (both $\Delta=0.4$ [0.0; $0.7]$, ES $=0.54) . P_{\max }\left(\mathrm{W} \cdot \mathrm{kg}^{-1}\right)$ was significantly higher in GII compared to GI $(\Delta=0.3[0.1 ; 0.5]$, ES = $0.73)$, GIII $(\Delta=0.2[0.1 ; 0.4], \mathrm{ES}=0.56)$ and GIV $(\Delta=$ $0.3[0.1 ; 0.5], \mathrm{ES}=0.76) . \mathrm{HR}_{\max }$ (beats. $\mathrm{min}^{-1}$ ) was significantly lower in Giv compared to GI $(\Delta=-15$ $[-21 ;-10]$, ES $=1.43)$, GII $(\Delta=-12[-17 ;-8]$, ES $=$ $1.17)$, and GIII $(\Delta=-11[-16 ;-5], \mathrm{ES}=1.01) . \mathrm{HR}_{\text {rest }}$ (beats $\left.\cdot \mathrm{min}^{-1}\right)$ was significantly lower in GII $(\Delta=-6$ $[-10 ;-1], \mathrm{ES}=0.57)$ and GIv $(\Delta=-6[-11$ to 0$], \mathrm{ES}=$ $0.57)$ compared to GI.

Based on the ANOVA results (Table 2), Ln $\mathrm{HF}_{\text {supine }}\left(\mathrm{ms}^{2}\right)$ was significantly lower in GIII $(\Delta=-$ $0.8[-1.4 ;-0.1], \mathrm{ES}=0.62)$ and GIV $(\Delta=-1.0[-1.6 ;-$ $0.3], \mathrm{ES}=0.76)$ compared to G. Also GiII $(\Delta=-0.6$ [$1.1 ; 0.0], \mathrm{ES}=0.46)$ and Giv $(\Delta=-0.8[-1.3 ;-0.2]$, ES $=0.61)$ were significantly lower than GII. Ln $\mathrm{LF} / \mathrm{HF}_{\text {supine values in } \mathrm{GIII}}(\Delta=1.0[0.2 ; 1.7], \mathrm{ES}=$ $0.66)$ and Giv $(\Delta=0.8[0.0 ; 1.6]$, ES $=0.53)$ were significantly higher than in Gi and $\mathrm{Ln} \mathrm{LF} / \mathrm{HF}_{\text {supine }}$ values in GIII $(\Delta=0.7[0.1 ; 1.4]$, ES $=0.50)$ were significantly higher than in GII. Ln Totsupine $\left(\mathrm{ms}^{2}\right)$ in GI $(\Delta=0.7[0.0 ; 1.3], \mathrm{ES}=0.57)$ and GII $(\Delta=0.6[0.0$; $1.1]$, ES $=0.48)$ was significantly higher compared to Giv. 
Table 1

Results of anthropometric and physiological variables in professional soccer players divided into four age groups

\begin{tabular}{|c|c|c|c|c|c|c|}
\hline \multirow[b]{2}{*}{ Variables (units) } & \multicolumn{4}{|c|}{ Age group } & \multirow[b]{2}{*}{$p$} & \multirow[b]{2}{*}{$\eta^{2}$} \\
\hline & GI & GII & GIII & GIV & & \\
\hline Number of players & 23 & 45 & 30 & 26 & & \\
\hline Age (years) & $18.6 \pm 0.8+\ddagger \S$ & $22.5 \pm 1.2^{*} \neq \S$ & $26.7 \pm 1.4^{*}+\S$ & $33.3 \pm 2.8^{*}+\ddagger$ & $<0.001$ & 0.90 \\
\hline BW (kg) & $73 \pm 6+\neq \S$ & $78 \pm 6^{*} \S$ & $79 \pm 8^{*}$ & $83 \pm 7^{*}+$ & $<0.001$ & 0.20 \\
\hline $\mathrm{BH}(\mathrm{cm})$ & $181 \pm 6$ & $183 \pm 5$ & $182 \pm 6$ & $186 \pm 7$ & 0.067 & 0.06 \\
\hline BMI $\left(\mathrm{kg} \cdot \mathrm{m}^{-2}\right)$ & $22.1 \pm 1.3+\neq \S$ & $23.4 \pm 1.4^{*}$ & $23.9 \pm 1.3^{*}$ & $24.1 \pm 1.1^{*}$ & $<0.001$ & 0.21 \\
\hline$\% \mathrm{BF}$ & $8.7 \pm 2.9 \pm \S$ & $10.2 \pm 2.6 \S$ & $11.4 \pm 2.2^{*}$ & $12.5 \pm 2.1^{*}+$ & $<0.001$ & 0.22 \\
\hline FFM (kg) & $66 \pm 5+\S$ & $70 \pm 5^{*}$ & $70 \pm 6$ & $73 \pm 6^{*}$ & 0.002 & 0.12 \\
\hline VT $\left(\% \mathrm{VO}_{2 \max }\right)$ & $82.1 \pm 2.0$ & $81.4 \pm 1.4$ & $81.9 \pm 1.4$ & $81.7 \pm 1.7$ & 0.273 & 0.03 \\
\hline $\mathrm{VO}_{2 \max }\left(\mathrm{ml} \cdot \mathrm{kg}^{-1} \cdot \mathrm{min}^{-1}\right)$ & $59.6 \pm 3.9 \S$ & $59.4 \pm 4.2 \S$ & $59.7 \pm 4.1 \S$ & $56.6 \pm 3.8^{*}+\ddagger$ & 0.015 & 0.08 \\
\hline $\mathrm{VO}_{2 \text { maxabs }}\left(1 \cdot \mathrm{min}^{-1}\right)$ & $4.3 \pm 0.5 \ddagger \S$ & $4.6 \pm 0.5$ & $4.7 \pm 0.4^{*}$ & $4.7 \pm 0.5^{*}$ & 0.018 & 0.08 \\
\hline$P_{\max }\left(W \cdot \mathrm{kg}^{-1}\right)$ & $6.3 \pm 0.3+$ & $6.6 \pm 0.3^{*} \mp \S$ & $6.3 \pm 0.3+$ & $6.3 \pm 0.3+$ & $<0.001$ & 0.19 \\
\hline $\mathrm{HR}_{\max }\left(\right.$ beats $\cdot \mathrm{min}^{-1}$ ) & $193 \pm 7 \S$ & $191 \pm 8 \S$ & $189 \pm 6 \S$ & $178 \pm 9 *+\ddagger$ & $<0.001$ & 0.34 \\
\hline
\end{tabular}

GI, 17-19.9 years; GII, 20-24.9 years; GIII, 25-29.9 years; GIV, 30-39 years; * statistically significant difference compared to $G_{1} ;$, statistically significant difference compared to GII; $\neq$,

statistically significant difference compared to GIII; $\S$, statistically significant difference compared to GIV; $p$, significance of ANOVA; $\eta^{2}$, eta-squared (ANOVA effect size); BW, body weight; $B H$, body height; $B M I$, body mass index; \%BF, percent of body fat; FFM, fat free mass; $V T$, anaerobic ventilatory threshold; $V O_{2 \max }$, maximal oxygen uptake expressed relative to $B W$;

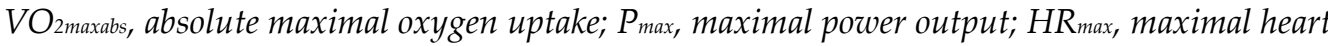
rate. Data are expressed as mean \pm standard deviation.

Table 2

Results of HRV variables in professional soccer players divided into four age groups

\begin{tabular}{|c|c|c|c|c|c|c|}
\hline \multirow[b]{2}{*}{ Variables (units) } & \multicolumn{4}{|c|}{ Age group } & \multirow[b]{2}{*}{$p$} & \multirow[b]{2}{*}{$\eta^{2}$} \\
\hline & GI & GII & GIII & GIV & & \\
\hline HRrest (beats·min ${ }^{-1}$ ) & $56 \pm 6+\S$ & $50 \pm 6^{*}$ & $52 \pm 9$ & $50 \pm 7^{*}$ & 0.013 & 0.09 \\
\hline Ln LF supine $\left(\mathrm{m} \cdot \mathrm{s}^{2}\right)$ & $6.4 \pm 0.8$ & $6.4 \pm 1.3$ & $6.6 \pm 1.1$ & $6.2 \pm 1.0$ & 0.652 & 0.01 \\
\hline Ln HF supine $\left(m \cdot s^{2}\right)$ & $7.9 \pm 0.6 \neq \S$ & $7.7 \pm 0.9 \ddagger \S$ & $7.1 \pm 0.8^{*} \dagger$ & $6.9 \pm 1.0^{*} \dagger$ & $<0.001$ & 0.16 \\
\hline Ln LF/HF supine & $-1.5 \pm 0.9 \ddagger \S$ & $-1.3 \pm 1.2 \ddagger$ & $-0.5 \pm 0.8^{*} \dagger$ & $-0.7 \pm 1.2^{*}$ & 0.002 & 0.12 \\
\hline Ln Totsupine $\left(\mathrm{m} \cdot \mathrm{s}^{2}\right)$ & $8.1 \pm 0.6 \S$ & $8.0 \pm 0.9 \S$ & $7.6 \pm 0.9$ & $7.4 \pm 0.9^{*}+$ & 0.009 & 0.09 \\
\hline Ln RMSSD supine $(\mathrm{m} \cdot \mathrm{s})$ & $4.6 \pm 0.3 \S$ & $4.6 \pm 0.4 \ddagger \S$ & $4.3 \pm 0.5 t$ & $4.2 \pm 0.5^{*} \dagger$ & 0.001 & 0.13 \\
\hline $\operatorname{Ln~LF}_{\text {standing }}\left(\mathrm{m} \cdot \mathrm{s}^{2}\right)$ & $6.5 \pm 1.0$ & $6.8 \pm 0.9$ & $6.7 \pm 0.9$ & $6.5 \pm 1.2$ & 0.402 & 0.02 \\
\hline Ln $\mathrm{HF}_{\text {standing }}\left(\mathrm{m} \cdot \mathrm{s}^{2}\right)$ & $5.3 \pm 1.0$ & $5.5 \pm 1.2$ & $5.1 \pm 1.3$ & $5.1 \pm 1.3$ & 0.469 & 0.02 \\
\hline Ln LF/HF standing & $1.3 \pm 0.6$ & $1.4 \pm 0.9$ & $1.7 \pm 1.1$ & $1.4 \pm 1.2$ & 0.487 & 0.02 \\
\hline Ln Totstanding $\left(\mathrm{m} \cdot \mathrm{s}^{2}\right)$ & $6.8 \pm 0.9$ & $7.1 \pm 0.9$ & $7.0 \pm 0.9$ & $6.8 \pm 1.1$ & 0.456 & 0.02 \\
\hline 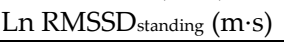 & $3.0 \pm 0.6$ & $3.3 \pm 0.6$ & $3.2 \pm 0.6$ & $3.2 \pm 0.6$ & 0.299 & 0.03 \\
\hline
\end{tabular}

GI, 17-19.9 years; GII, 20-24.9 years; GIII, 25-29.9 years; GIV, 30-39 years; *, statistically significant difference compared to $\mathrm{G}_{\mathrm{I}} ; t$, statistically significant difference compared to GII; $\neq$, statistically significant difference compared to GIII; , statistically significant difference compared to GIV; $p$, significance of ANOVA; $\eta^{2}$, eta-squared ( $A N O V A$ effect size); HR rest, resting heart rate in the supine position; Ln LF, natural logarithm of low-frequency power; Ln

$H F$, natural logarithm of high-frequency power; Ln LF/HF, natural logarithm of the lowfrequency/high-frequency ratio; Ln Tot, natural logarithm of total power ( $L F+H F)$; Ln RMSSD, natural logarithm of root mean square successive difference of RR intervals; supine, supine position in the orthoclinostatic maneuver; standing, standing position in the orthoclinostatic maneuver. Data are expressed as mean \pm standard deviation. 
Ln RMSSD supine $(\mathrm{ms})$ in GIII $(\Delta=-0.3[-0.6 ; 0.0]$, $\mathrm{ES}=0.47)$ and GIv $(\Delta=-0.4[-0.7 ;-0.1], \mathrm{ES}=0.57)$ was significantly lower than in GII and GIv $(\Delta=-$ $0.4[-0.7 ; 0.0], \mathrm{ES}=0.59)$ was significantly lower than G. Correlation analysis revealed a significant relationship between age and supine $\mathrm{HRV}$ variables as follows: Ln HF ( $\mathrm{r}=-0.41 ; p<0.001)$, Ln RMSSD ( $\mathrm{r}=-0.36 ; p<0.001)$ and Ln LF/HF ( $\mathrm{r}=$ $0.27 ; p=0.002)$. A significant correlation between $\%$ BF and Ln LF/HF ( $\mathrm{r}=0.27, p=0.002)$ and Ln HF $(\mathrm{r}=-0.20, p=0.028)$ was also found.

\section{Discussion}

The main aim of this study was to compare the somatic, endurance performance determinants, and HRV profiles of professional soccer players divided into different age groups. The main findings were that i) VO2max was significantly lower in the oldest players (30-39 years) compared to all the younger age groups, while the VT level was similar across the agegroups, ii) GII players (20.0-24.9 years) achieved the greatest Pmax values, whereas Pmax was similar across the other age groups, iii) the greatest BW was found in the oldest players most likely because of a greater \%BF and FFM, iv) significantly decreased basal vagal activity accompanied by a small increase in sympathetic activity was observed in players over 25 years of age.

In the present study, the players, excluding goalkeepers, achieved mean VO2max of $59.2 \pm 4.1 \mathrm{ml} \bullet \mathrm{kg}-1 \bullet \mathrm{min}-1$. This is comparable with the aerobic fitness levels of professional soccer players reported by Stølen et al. (2005). A slightly higher aerobic capacity was indicated by Tønnessen et al. (2013) who reported values of $\sim 62-64 \mathrm{ml} \bullet \mathrm{min}-1 \bullet \mathrm{kg}-1$ for professional soccer players in Norway. Our results show that players aged $>30$ years exhibit a significant decrease in both $\mathrm{VO} 2 \max (5 \% \sim 3 \mathrm{ml} \bullet \mathrm{kg}-1 \bullet \min -1)$ and the HRmax $(5.8 \%)$ compared to the younger agegroups. Hawkins and Wiswell (2003) reported that reductions in both HRmax and muscle mass were considered to be the primary factors responsible for the decrement in VO2max with age. Although lower VO2max values were found in our oldest players, they achieved greater VO2maxabs and FFM values compared to the younger players. According to Perim et al. (2011), a decline in the HRmax in endurance trained athletes may be compensated for by an increase in stroke volume to ensure optimal cardiac output. Therefore, we suggest that the reduction in the HRmax did not induce notable changes in VO2max levels in our oldest players. In contrast to our findings, Signorelli et al. (2012) who also assessed differences in pre-season VO2max in elite Brazilian soccer players, reported no significant differences in VO2max between younger (17-22 years) and older (27-36 years) players. However, there were no differences in BW between their age groups compared with our oldest players who were approximately $4 \mathrm{~kg}$ heavier than the younger age groups. Therefore, it is possible that the increase in BW contributed to the decrease in relative values of $\mathrm{VO} 2 \mathrm{max}$ in our players over the age of 30 years. On the other hand, the decline in VO2max in the oldest players was not followed by deteriorations in either the VT level or Pmax compared to the younger agegroups. We assume that the gain in BW, particularly in FFM, likely contributed to this finding. Previously, strength training and associated increases in FFM had been shown to increase Pmax and in turn work economy (Balsalobre-Fernández et al., 2015; Hoff and Helgerud, 2004). Whether strength training, gains in FFM and the associated maintenance of Pmax in the oldest players contributed to their performance requires further investigation.

In the present study, a resting HR was 6 beats $\bullet$ min- 1 lower in the oldest players compared to the youngest players. However, players $>30$ years exhibited significantly lower vagal activity compared to younger players. A discrepancy between resting HR values and the corresponding vagal activity was previously reported in well trained athletes in a meta-analysis by Sandercock et al. (2005). These authors suggested that increased vagal modulation was responsible for the initial reduction in a resting $H R$, and other factors such as changes in heart geometry and/or a reduction in an intrinsic HR may be responsible for further decreases in a resting HR with ageing without further changes in HRV.

In the present study, supine Ln HF and Ln RMSSD, both thought to reflect cardiac vagal activity (Buchheit, 2014; Task Force, 1996), were negatively correlated with age. In addition, a reduction in vagal activity induced a small relative increase in sympathetic activity in players 
over the age of 25 years. Our results are in agreement with Antelmi et al. (2004) who found a significant decrease in vagal activity (represented by $\mathrm{HF}$ and RMSSD) and an increase in the LF/HF ratio in each decade from the junior age $(<19$ years) until the fourth and the sixth decade of life. In addition to aging, the increase in \%BF could be another variable that had a negative effect on autonomic cardiac control. In this regard, Christou et al. (2004) reported that increased adiposity with advancing age was related to impairment of autonomic cardiac associated with cardiac electrical instability, increased susceptibility to ventricular tachyarrhythmias and an increased incidence of cardiac sudden death in the presence of myocardial ischemia (Billman, 2009; Schwartz et al., 1988).

Frick (2007) reported that the playing careers of professional soccer players were short ( 4 years), with a minority of all careers exceeding 9 years. During their careers professional players experience high amounts of psychological and physical stress (typically training 6 times a week with 1-2 matches per week). The psychological and physical stress contributes to the allostatic load experienced by players. The allostatic load is thought to result in detrimental physiological consequences as a result of chronic exposure to fluctuating or heightened neural or neuroendocrine responses (hypothalamic-pituitary-adrenal axis) that also induce a reduction in vagal activity (Thayer and Sternberg, 2006). For instance, Iellamo et al. (2002) showed that very intensive endurance training shifted the cardiovascular autonomic modulation towards sympathetic dominance with a concomitant increase in blood pressure. The authors suggested whether altered blood pressure and autonomic function might in time be hazardous to the cardiovascular system in athletes. Based on this discussion, it is tenable that age (specifically professional career length duration), psychological and physical stress as well as a \%BF level in soccer players could be considered co-factors that contribute to vagal cardiac deterioration. In this regard, specific interventions could be used to reduce the allostatic load and the consequent detrimental effects on autonomic cardiac control in specific players e.g. a nutritional intervention to reduce $\% \mathrm{BF}$, or yoga to manage chronic stress. Patil et al.
(2013) reported that yoga practice helped to reduce mental stress associated with chronic training in cyclists and optimized autonomic cardiac function. In addition to the above factors, attenuated vagal activity is related to intensive blocks of training (Pichot et al., 2000) and is significantly associated with weaker adaptation responses to both aerobic (Botek et al., 2014a; Buchheit, 2014; Hautala et al., 2009; Vesterinen et al., 2015) and anaerobic training (Boutcher et al., 2013). Based on our findings, we propose the implementation of regular HRV monitoring during training in professional soccer players as it provides important diagnostic information about changes in autonomic cardiac regulation over their careers. Such information could be of benefit for adjusting training volume and/or recovery strategies, especially in players who exhibit lower cardiac vagal control.

To conclude, $\mathrm{VO} 2 \mathrm{max}$ is relatively stable $(\sim 59 \mathrm{ml} \bullet \mathrm{kg}-1 \bullet \mathrm{min}-1)$ in professional soccer players up to 30 years of age. It then decreases by $\sim 5 \%(\sim 3 \mathrm{ml} \bullet \mathrm{kg}-1 \bullet \mathrm{min}-1)$ in following nine years possibly due to a gain in BW. Although a decline in VO2max occurred, players $>25$ years of age demonstrated similar Pmax levels. Regarding the autonomic cardiac profile, the first decline in vagal activity with a minor relative increase in sympathetic activity was demonstrated in players $>25$ years of age. It should be highlighted that knowledge of the age-related changes in body composition, autonomic cardiac regulation and a physiological profile of professional soccer players throughout their career provides an opportunity for coaches to change their training approach in order to enhance and/or maintain fitness performance, particularly in teams, where marked age-heterogeneity in players is present.

\section{Study limitations}

Professional soccer players usually perform pre-season laboratory testing after a 3-4 week transitory period. In the Czech Republic, typical individually based training programs during this period involve combinations of continual running, resistant training, and additional sports events such as foot tennis and/or swimming. However, the training load, together with dietary habits, may vary among soccer players. This variation in training loads and dietary intake is considered a main limitation of the study, as it could affect the current player's 
profile before laboratory testing. As reported by Sotiropoulos et al. (2009), a four week transition period in professional soccer players using a traditional program led to a reduction in $\mathrm{VO} 2 \mathrm{max}$ accompanied with a greater gain in $\mathrm{BW}$ and $\% \mathrm{BF}$ compared to players who followed specific training drills.

\section{Acknowledgement}

This work was supported by the Faculty of Physical Culture under Grant IGA_FTK_2015_005.

\section{References}

Akselrod S, Gordon D, Ubel FA, Shannon DC, Berger AC, Cohen RJ. Power spectrum analysis of heart rate fluctuation: a quantitative probe of beat-to-beat cardiovascular control. Science, 1981; 213: 220-222

American College of Sports Medicine. Guidelines for exercise testing and prescription. 3rd ed. Philadelphia, PA: Lea \& Febiger; 1986

Antelmi I, de Paula RS, Shinzato AR, Peres CA, Mansur AJ, Grupi CJ. Influence of age, gender, body mass index, and functional capacity on heart rate variability in a cohort of subjects without heart disease. Am J Cardiol, 2004; 93: 381-385

Arroyo M, Gonzalez-de-Suso JM, Sanchez C, Ansotegui L, Rocandio AM. Body image and body composition: comparisons of young male elite soccer players and controls. Int J Sport Nutr Exerc Metab, 2008; 18: 628-638

Aubert AE, Seps B, Beckers F. Heart rate variability in athletes. Sports Med, 2003; 33: 889-919

Balsalobre-Fernández C, Santos-Concejero J, Grivas GV. The effects of strength training on running economy in highly trained runners: a systematic review with meta-analysis of controlled trials. J Strength Cond Res, 2015 [Epub ahead of print]

Banach T, Zoładź JA, Kolasińska-Kloch W, Szyguła Z, Thor PJ. The effect of aging on the activity of the autonomic nervous system in long distance runners. Folia Med Cracov, 2000; 43: 113-120

Billman GE. Cardiac autonomic neural remodeling and susceptibility to sudden cardiac death: effect of endurance exercise training. Am J Physiol Heart Circ Physiol, 2009; 297: H1171-H1193

Botek M, Krejčí J, Neuls F, Novotný J. Effect of modified method of autonomic nervous system activity assessment on results of heart rate variability analysis. Acta Univ Palacki Olomuc Gymn, 2013; 43: 39-46

Botek M, Krejčí J, Weisser R. Autonomic cardiac regulation and morpho-physiological responses to eight week training preparation in junior soccer players. Acta Gymnica, 2014a; 44: 155-163

Botek M, McKune AJ, Krejci J, Stejskal P, Gaba A. Change in performance in response to training load adjustment based on autonomic activity. Int J Sports Med, 2014b; 35: 482-488

Boutcher SH, Park Y, Dunn SL, Boutcher YN. The relationship between cardiac autonomic function and maximal oxygen uptake response to high-intensity intermittent-exercise training. J Sports Sci, 2013; 31: 1024-1029

Brooks GA, Fahey TD, Baldwin KM. Exercise Physiology: Human Bioenergetics and Its Applications. $4^{\text {th }}$ ed. London, United Kingdom: McGraw-Hill Education; 2004

Buchheit M. Monitoring training status with HR measures: do all roads lead to Rome? Front Physiol, 2014; 5: 73

Christou DD, Jones PP, Pimentel AE, Seals DR. Increased abdominal-to-peripheral fat distribution contributes to altered autonomic-circulatory control with human aging. Am J Physiol Heart Circ Physiol, 2004; 287: H1530-H1537

De Meersman RE, Stein PK. Vagal modulation and aging. Biol Psychol, 2007; 74: 165-173 
Eckberg DL. Sympathovagal balance: a critical appraisal. Circulation, 1997; 96: 3224-3232

Frick B. The football players' labor market: empirical evidence from the major European leagues. Scott J Polit Econ, 2007; 54: 422-446

Gil SM, Gil J, Ruiz F, Irazusta A, Irazusta J. Physiological and anthropometric characteristics of young soccer players according to their playing position: relevance for the selection process. J Strength Cond Res, 2007; 21: 438-445

Hautala AJ, Kiviniemi AM, Tulppo MP. Individual responses to aerobic exercise: the role of the autonomic nervous system. Neurosci Biobehav Rev, 2009; 33: 107-115

Hawkins S, Wiswell R. Rate and mechanism of maximal oxygen consumption decline with aging: implications for exercise training. Sports Med, 2003; 33: 877-888

Helgerud J. Maximal oxygen uptake, anaerobic threshold and running economy in women and men with similar performances level in marathons. Eur J Appl Physiol Occup Physiol, 1994; 68: 155-161

Helgerud J, Rodas G, Kemi OJ, Hoff J. Strength and endurance in elite football players. Int J Sports Med, 2011; 32: $677-682$

Hoff J, Helgerud J. Endurance and strength training for soccer players: physiological considerations. Sports Med, 2004; 34: 165-180

Hopkins WG. Measures of reliability in sports medicine and science. Sports Med, 2000; 30: 1-15

Hopkins WG, Marshall SW, Batterham AM, Hanin J. Progressive statistics for studies in sports medicine and exercise science. Med Sci Sports Exerc, 2009; 41: 3-13

Iellamo F, Legramante JM, Pigozzi F, Spataro A, Norbiato G, Lucini D, Pagani M. Conversion from vagal to sympathetic predominance with strenuous training in high-performance world class athletes. Circulation, 2002; 105: 2719-2724

Malik M, Camm AJ. Heart rate variability. New York, NY: Futura Publishing; 1995

Malliani A, Pagani M, Lombardi F, Cerutti S. Cardiovascular neural regulation explored in the frequency domain. Circulation, 1991; 84: 482-492

Millet GP, Libicz S, Borrani F, Fattori P, Bignet F, Candau R. Effects of increased intensity of intermittent training in runners with differing $\mathrm{VO}_{2}$ kinetics. Eur J Appl Physiol, 2003; 90: 50-57

Mølgaard H, Sørensen KE, Bjerregaard P. Circadian variation and influence of risk factors on heart rate variability in healthy subjects. Am J Cardiol, 1991; 68: 777-784

Pate RR, Kriska A. Physiological basis of the sex difference in cardiorespiratory endurance. Sports Med, 1984; 1: $87-98$

Patil SG, Mullur LM, Khodnapur JP, Dhanakshirur GB, Aithala MR. Effect of yoga on short-term heart rate variability measure as a stress index in subjunior cyclists: a pilot study. Indian J Physiol Pharmacol, 2013; 57: 153-158

Penttilä J, Helminen A, Jartti T, Kuusela T, Huikuri HV, Tulppo MP, Coffeng R, Scheinin H. Time domain, geometrical and frequency domain analysis of cardiac vagal outflow: effects of various respiratory patterns. Clin Physiol, 2001; 21: 365-376

Perim RR, Signorelli GR, Myers J, Arena R, de Araújo CG. The slope of the oxygen pulse curve does not depend on the maximal heart rate in elite soccer players. Clinics, 2011; 66: 829-835

Pichot V, Roche F, Gaspoz JM, Enjolras F, Antoniadis A, Minini P, Costes F, Busso T, Lacour JR, Barthélémy JC. Relation between heart rate variability and training load in middle-distance runners. Med Sci Sports Exerc, 2000; 32: 1729-1736

Pimentel AE, Gentile CL, Tanaka H, Seals DR, Gates PE. Greater rate of decline in maximal aerobic capacity with age in endurance-trained than in sedentary men. J Appl Physiol, 2003; 94: 2406-2413

Rampinini E, Impellizzeri FM, Castagna C, Abt G, Chamari K, Sassi A, Marcora SM. Factors influencing 
physiological responses to small-sided soccer games. J Sports Sci, 2007; 25: 659-666

Reilly T, Williams AM. Science and Soccer. $2^{\text {nd }}$ ed. London, United Kingdom: Routledge; 2003

Sandercock GR, Bromley PD, Brodie DA. Effects of exercise on heart rate variability: inferences from metaanalysis. Med Sci Sports Exerc, 2005; 37: 433-439

Schwartz PJ, Vanoli E, Stramba-Badiale M, De Ferrari GM, Billman GE, Foreman RD. Autonomic mechanisms and sudden death. New insights from analysis of baroreceptor reflexes in conscious dogs with and without a myocardial infarction. Circulation, 1988; 78: 969-979

Shephard RJ, Åstrand PO. Endurance in sport. Oxford, UK: Blackwell Science, 192-193; 1992

Signorelli GR, Perim RR, Santos TM, Araujo CG. A pre-season comparison of aerobic fitness and flexibility of younger and older professional soccer players. Int J Sports Med, 2012; 33: 867-872

Sotiropoulos A, Travlos AK, Gissis I, Souglis AG, Grezios A. The effect of a 4-week training regimen on body fat and aerobic capacity of professional soccer players during the transition period. J Strength Cond Res, 2009; 23: 1697-1703

Stølen T, Chamari K, Castagna C, Wisløff U. Physiology of soccer: an update. Sports Med, 2005; 35: 501-536

Task Force of the European Society of Cardiology and the North American Society of Pacing and Electrophysiology. Heart rate variability. Standards of measurement, physiological interpretation and clinical use. Eur Heart J, 1996; 17: 354-381

Tessitore A, Meeusen R, Tiberi M, Cortis C, Pagano R, Capranica L. Aerobic and anaerobic profiles, heart rate and match analysis in older soccer players. Ergonomics, 2005; 15: 1365-1377

Thayer JF, Sternberg E. Beyond heart rate variability: vagal regulation of allostatic systems. Ann N Y Acad Sci, 2006; 1088: 361-372

Tønnessen E, Hem E, Leirstein S, Haugen T, Seiler S. Maximal aerobic power characteristics of male professional soccer players, 1989-2012. Int J Sports Physiol Perform, 2013; 8: 323-329

Trappe SW, Costill DL, Vukovich MD, Jones J, Melham T. Aging among elite distance runners: a 22-yr longitudinal study. J Appl Physiol, 1996; 80: 285-290

Vanoli E, De Ferrari GM, Stramba-Badiale M, Hull SS Jr, Foreman RD, Schwartz PJ. Vagal stimulation and prevention of sudden death in conscious dogs with a healed myocardial infarction. Circ Res, 1991; 68: 1471-1481

Vesterinen V, Häkkinen K, Laine T, Hynynen E, Mikkola J, Nummela A. Predictors of individual adaptation to high-volume or high-intensity endurance training in recreational endurance runners. Scand J Med Sci Sports, 2015; doi: 10.1111/sms.12530 [Epub ahead of print]

Wasserman K, Hansen JE, Sue DY, Whipp BJ. Principles of exercise testing and interpretation. Philadelphia, PA: Lea \& Febiger, 1987

Yamamoto Y, Hughson RL. Coarse-graining spectral analysis: new method for studying heart rate variability. J Appl Physiol, 1991; 71: 1143-1150

\section{Corresponding author:}

\section{Jakub Krejčí, PhD}

Department of Natural Sciences in Kinanthropology

Faculty of Physical Culture, Palacký University Olomouc

třída Míru 117, 77111 Olomouc, Czech Republic

Phone: +420 585636414

Fax: +420 585412899

E-mail: jakub.krejci@upol.cz 